\title{
A Note on the Homology of Signed Posets
}

\author{
PHIL HANLON* \\ Department of Mathematics, University of Michigan, Ann Arbor, MI 48109-1003
}

Received February 7,1994; Revised May 17, 1995

\begin{abstract}
Let $S$ be a signed poset in the sense of Reiner [4]. Fischer [2] defines the homology of $S$, in terms of a partial ordering $P(S)$ associated to $S$, to be the homology of a certain subcomplex of the chain complex of $P(S)$.

In this paper we show that if $P(S)$ is Cohen-Macaulay and $S$ has rank $n$, then the homology of $S$ vanishes for degrees outside the interval $[n / 2, n]$.
\end{abstract}

Keywords: poset, Cohen-Macaulay, signed poset

\section{Introduction}

Let $R$ be a set of vectors in $\mathbb{R}^{n}$. The positive linear closure of $R$, denoted $\bar{R}$ is defined to be the span of all linear combinations of vectors in $R$ with non-negative real coefficients.

For each $i=1,2, \ldots, n$ let $e_{i}$ denote the $i$ th unit coordinate vector in $\mathbb{R}^{n}$ and let $e_{-i}$ denote $-e_{i}$. Recall that the root system $B_{n}$ is the set

$$
B_{n}=\left\{ \pm\left(e_{i} \pm e_{j}\right): 1 \leq i<j \leq n\right\} \cup\left\{ \pm e_{i}: 1 \leq i \leq n\right\}
$$

Definition 1 A signed poset is a subset $S$ of $B_{n}$ such that

(a) $S \cap(-S)=\emptyset$.

(b) $\bar{S} \cap B_{n}=S$.

Let $(P, \leq)$ be an ordinary poset with $P=\{1,2, \ldots, n\}$. Let $S$ be the collection of all $e_{i}-e_{j}$ such that $i<j$. Then $S$ is a subset of the root system $A_{n}$ which satisfies conditions (a) and (b) of Definition 1 (where $B_{n}$ is replaced by $A_{n}$ in condition (b)). Vic Reiner introduced the notion of signed poset [4] to be a $B_{n}$-analogue of the notion of poset.

In more recent work Steve Fischer [2] defined a homology theory for signed posets. According to Fischer's definition, the homology of a signed poset $S$ is the homology of a certain simplicial complex $C_{*}^{0}(S)$ associated to $S$. This simplicial complex is analogous to the simplicial complex of chains in a poset. Fischer showed that the Euler characteristic of this homology can be computed via a "2-Mobius function" and that analogues of Weisner's Theorem and Crapo's Complementation Theorem can be used to calculate this 2-Mobius

${ }^{*}$ Research partially supported by the National Science Foundation and the John Simon Guggenheim Foundation. 
function when $S$ is a "signed lattice". In view of these results on the 2-Mobius function, it would be interesting to know if there are combinatorial labelling conditions which would imply that the simplicial complex associated to $S$ is shellable.

There is an obvious analogue of EL-labelling that can be defined for signed posets, namely we say that $S$ is EL-labellable iff $P(S)$ is EL-labellable. Here $P(S)$ is a poset whose chains are used to define $C_{*}^{0}(S)$. An EL-labellable signed poset is pure in the sense that all facets of $C_{*}^{0}(S)$ have the same dimension (which we will call the dimension of $S$ ). Originally Fischer had hoped to show that if $S$ is EL-labellable then the homology of $C_{*}^{0}(S)$ is zero except in the top dimension. But then he constructed two EL-labellable signed posets $S_{0}$ and $S_{1}$ such that

(a) the homology of $C_{*}^{0}\left(S_{0}\right)$ is nonzero exactly in degree equal to half the top dimension.

(b) the homology of $C_{*}^{0}\left(S_{1}\right)$ is nonzero exactly in degree equal to the top dimension.

He went on to define "signed EL-labelling" to be an EL-labelling that satisfies other conditions and showed that the existence of a signed EL-labelling of $S$ implies that $C_{*}^{0}(S)$ is shellable.

The purpose of this note is to prove that the examples $S_{0}$ and $S_{1}$ above are the extreme cases, i.e., we will prove.

Theorem 1 Suppose $S$ is an EL-labellable signed poset of dimension n. Then $H_{r}(S)$ is 0 unless $\lfloor n / 2\rfloor \leq r \leq n$.

\section{Homology of a signed poset}

We begin this section by defining the simplicial complex $C_{*}^{0}(S)$ that Fischer uses to compute the homology of $S$. This complex is given in terms of the chains in a certain poset $P(S)$.

Definition 2 Let $S$ be a signed poset in $B_{n}$. Define the poset $P(S)$ with vertex set $\{ \pm 1, \ldots, \pm n\}=V$ as follows. For $u, v \in V$ we say

$$
u \leq P(S) v
$$

if and only if

(i) $e_{u}-e_{v} \in S \quad$ for $|u| \neq|v|$ or

(ii) $e_{u} \in S$ for $v=-u$.

Fischer showed that $P(S)$ is a self dual poset.

Definition 3 An isotropic $r$-chain in $P(S)$ is an $r$-chain

$$
\alpha_{1}<\alpha_{2}<\cdots<\alpha_{r}
$$

such that $\alpha_{i}$ is not equal to $-\alpha_{j}$ for any $i, j$. Let $\Delta_{r}^{0}(S)$ denote the collection of isotropic $r$-chains in $P(S)$ and let $C_{r}^{0}(S)$ denote the $\mathbb{C}$-span of $\Delta_{r}^{0}(S)$ (with $C_{0}^{0}(S)=\mathbb{C}$ ). 
Note that $\Delta_{r}^{0}(S)$ is a simplicial complex in $2^{P(S)}$. This gives a boundary map $\partial_{r}: C_{r}^{0}(S) \rightarrow$ $C_{r-1}^{0}(S)$,

$$
\partial_{r}\left(\alpha_{1}<\alpha_{2}<\cdots<\alpha_{r}\right)=\sum_{i=1}^{r}(-1)^{i-1}\left(\alpha_{1}<\cdots<\hat{\alpha}_{i}<\cdots<\alpha_{r}\right)
$$

Definition 4 Define $H_{r}^{0}(S)$ to be the $r$ th homology of the complex $\left(C_{*}^{0}(S), \partial_{*}\right)$, i.e.

$$
H_{r}^{0}(S)=\operatorname{ker} \partial_{r} / \mathrm{im} \partial_{r+1} \text {. }
$$

We call $H_{*}^{0}(S)$ the signed poset homology of $S$.

We say $S$ is EL-labellable if $P(S)$ has an EL-labelling. In [2], Fischer computes $C_{*}^{0}(S)$ and $H_{*}^{0}(S)$ for a number of signed posets $S$. In particular he constructs a family of posets $\Gamma_{n} \subseteq B_{n}$ such that:

- $\Delta^{0}\left(\Gamma_{n}\right)$ is pure of dimension $n$

- $\Gamma_{n}$ is EL-labellable

- $\Delta^{0}\left(\Gamma_{n}\right)$ is homotopic to the $\lfloor n / 2\rfloor$-dimensional sphere.

This family of signed posets shows that an EL-labelling on $S$ does not imply that $\Delta^{0}(S)$ is shellable.

\section{The main result}

Let $Q$ be a finite, ranked, self-dual poset. Let $x \rightarrow x^{*}$ be a fixed order-reversing involution on $Q$. Split $Q=Q^{L} \cup Q^{U}$ so that $Q^{L}$ is an order ideal in $Q,\left(Q^{L}\right)^{*} \cap Q^{L}=\{x \in Q$ : $\left.x^{*}=x\right\}$ and $\left(Q^{U}\right)^{*} \subseteq Q^{L}$. For each chain $\gamma=\alpha_{1}<\alpha_{2}<\cdots<\alpha_{r}$ define $\omega(\gamma)$ to be the number of pairs $\left(\alpha_{i}, \alpha_{j}\right)$ with $i<j$ and $\alpha_{j}=\alpha_{i}^{*}$. We say $\gamma$ is isotropic if $\omega(\gamma)=0$.

Let $C_{r}(Q)$ denote the span of all $r$-chains and $C_{*}^{0}(Q)$ the span of all isotropic $r$-chains. The boundary map $\partial_{*}: C_{*}(Q) \rightarrow C_{*-1}(Q)$ preserves $C_{*}^{0}(Q)$ and so $\left(C_{*}^{0}(Q), \partial_{*}\right)$ is a subcomplex of $\left(C_{*}(Q), \partial_{*}\right)$. Let $H_{*}^{0}(Q)$ denote the homology of that subcomplex. The main theorem for this section is:

Theorem 2 Suppose $Q$ is Cohen-Macaulay of rank $n$. Then

$$
H_{d}^{0}(Q)=0 \quad \text { unless } \frac{n}{2} \leq d \leq n
$$

Proof: We prove this by induction on $|Q|$. If $Q$ is the empty poset then $H_{d}^{0}(Q)$ is 0 unless $d=0$. This agrees with the statement in Theorem 2 since $n=0$ in this case.

Consider an arbitrary $Q$ and assume the result is true for all $Q^{\prime}$ with $\left|Q^{\prime}\right|<|Q|$.

Let $\gamma$ be a chain in $C_{*}(Q)$. We assign a non-negative integer $\rho(\gamma)$ to $\gamma$ as follows: 
1) If $\gamma$ is isotropic then $\rho(\gamma)=0$.

2) If $\gamma$ is not isotropic, write $\gamma$ as

$$
\alpha_{1}<\alpha_{2}<\cdots<\alpha_{r} .
$$

Then $\rho(\gamma)$ is the rank of $\alpha_{i}$ where $i$ is maximal subject to the condition that $\alpha_{i}^{*}=\alpha_{j}$ for some $j>i$. We also write $A(\gamma)$ to denote $\alpha_{i}$. Note that $A(\gamma) \in Q^{L}$.

For $r, p \in \mathbb{N}$ let $C_{r, p}(Q)$ denote the span of all $r$-chains $\gamma$ with $\rho(\gamma)=p$. Note that the boundary map $\partial$ satisfies:

$$
\partial\left(C_{r, p}(Q)\right) \subseteq \bigoplus_{t \leq p} C_{r-1, t}(Q)
$$

Thus $\left(C_{*}(Q), \partial\right)$ is filtered by the parameter $\rho$. Let $\left(E^{s}, \partial^{s}\right)$ be the associated spectral sequence which abutts to $E^{\infty}=H_{*}(Q)$. Background material on spectral sequences can be found in any introductory text in homological algebra (e.g. [1] or [3]).

Our first step will be to compute the $E^{1}$ term in this spectral sequence.

$E^{0}$ is the associated graded complex. Let $\gamma$ be an $r$-chain in $E_{r}^{0}$. Write $\gamma$ as

$$
\gamma=\alpha_{1}<\alpha_{2}<\cdots<\alpha_{i}<\alpha_{i+1}<\cdots<\alpha_{j-1}<\alpha_{j}=\alpha_{i}^{*}<\alpha_{j+1}<\cdots<\alpha_{r} .
$$

Then

$$
\partial^{0} \gamma=\sum_{s=1, s \neq i, j}^{r}(-1)^{s-1}\left(\alpha_{1}<\cdots<\hat{\alpha}_{s}<\cdots<\alpha_{r}\right) .
$$

Let $E_{r}^{0}[\alpha]$ denote the span of all $r$-chains $\gamma$ with $A(\gamma)=\alpha$ and let $E_{r}^{0}[0 \hat{]}]$ denote $C_{r}^{0}(Q)$. Then

1) $E_{r}^{0}=C_{r}^{0}(Q) \oplus \bigoplus_{\alpha \in Q^{L} \backslash(\hat{0}\}} E_{r}^{0}[\alpha]$

2) $\partial^{0}\left(E_{r}^{0}[\alpha]\right) \subseteq E_{r-1}^{0}[\alpha] \quad$ for all $\alpha \in Q^{L} \cup\{\hat{0}\}$.

So the complex $\left(E_{r}^{0}, \partial^{0}\right)$ splits as a direct sum of the subcomplexes

$$
\bigoplus_{\alpha \in Q^{L} \backslash(\hat{0})}\left(E_{*}^{0}[\alpha], \partial^{0}\right)
$$

We now analyze the subcomplex $\left(E_{*}^{0}[\alpha], \partial^{0}\right)$. Assume $\alpha \in Q^{L}$ and that $\alpha^{*}>\alpha$. For a chain $\gamma$ to have $A(\gamma)=\alpha$, it is necessary and sufficient for $\gamma$ to consist of any chain up to $\alpha$, then an isotropic chain $\alpha$ to $\alpha^{*}$, and then any chain from $\alpha^{*}$ upward. So,

$$
E_{*+2}^{0}[\alpha] \cong C_{*}\left(I_{\alpha}\right) \otimes C_{*}^{0}\left(\left(\alpha, \alpha^{*}\right)\right) \otimes C_{*}\left(I^{\alpha^{*}}\right)
$$

where $I_{\alpha}$ denotes the open order ideal generated by $\alpha$ in $Q, I^{\alpha^{*}}$ denotes the open order filter generated by $\alpha^{*}$ in $Q$ and $\left(\alpha, \alpha^{*}\right)$ is the open interval from $\alpha$ to $\alpha^{*}$ in $Q$. Moreover, (1) 


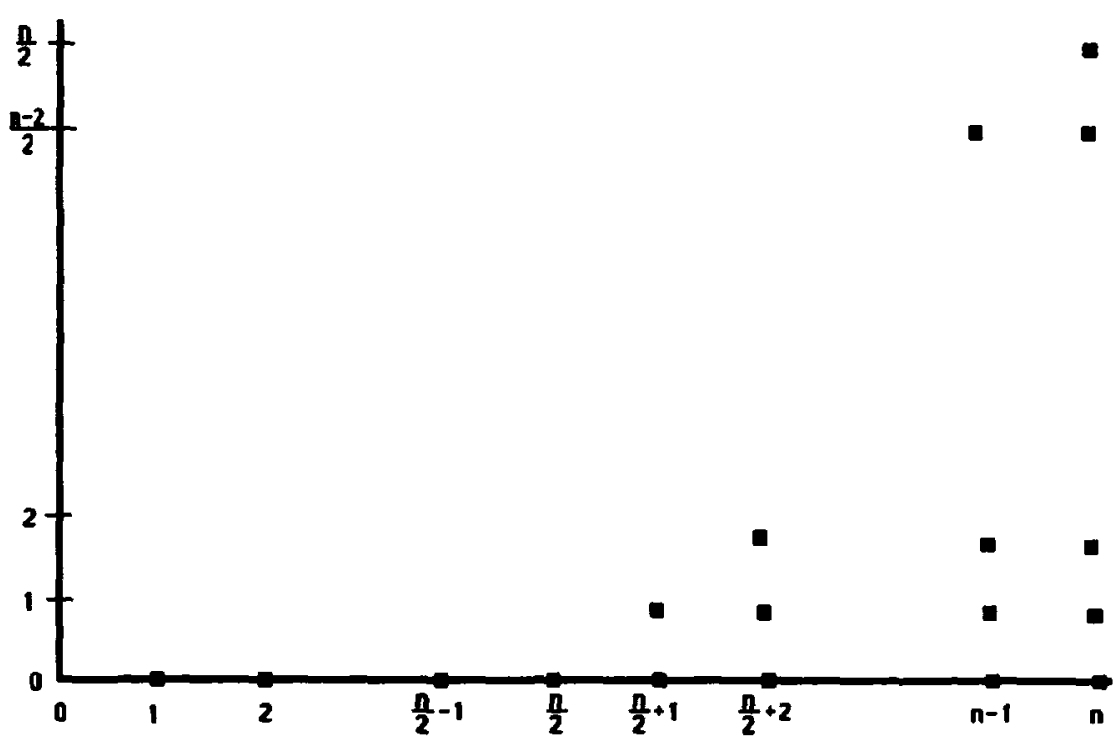

Figure 1 .

shows that the tensor product of vector spaces given by (2) extends to a tensor product of complexes.

Let $p$ be the rank of $\alpha$ so the rank of $\alpha^{*}$ is $n+1-p$. Since $Q$ is Cohen-Macaulay we have

$$
H_{d}\left(I_{\alpha}\right)=H_{d}\left(I^{\alpha^{*}}\right)=0 \quad \text { unless } d=p-1
$$

The self-dual poset $\left(\alpha, \alpha^{*}\right)$ is Cohen-Macaulay of rank $(n-p)-p=n-2 p$. By our induction hypothesis

$$
H_{d}^{0}\left(\left(\alpha, \alpha^{*}\right)\right)=0 \quad \text { unless } \frac{n-2 p}{2} \leq d \leq n-2 p .
$$

Combining these observations we find:

$$
E_{d}^{1}[\alpha]=0 \quad \text { unless } \frac{n}{2}+p \leq d \leq n .
$$

At this point we know nothing about

$$
E_{*}^{1}[\hat{0}]=\text { Homology of }\left(C_{*}^{0}(Q), \partial\right)=H_{*}^{0}(Q) .
$$

However we can draw a diagram of $E_{r, p}^{1}$ letting a square box denote values of $r, p$ where $E_{r, p}^{1}$ might be non-zero. This appears in Figure 1. 
The $\partial^{1}$ differential on $E^{1}$ maps $E_{r, p}^{1}$ to $E_{r-1, p-1}^{1}$. More generally, the $\partial^{s}$ differential on $E^{s}$ maps $E_{r, p}^{s}$ to $E_{r-1, p-s}^{s}$. It follows by induction on $s$ that

$$
E_{r, 0}^{s}=E_{r, 0}^{1}=E_{r}^{1}[\hat{0}]=H_{r}^{0}(Q)
$$

for $0 \leq r<\frac{n}{2}$ and all $s$. Thus

$$
H_{r}^{0}(Q)=E_{r, 0}^{\infty} \subseteq H_{r}(Q)=0 \quad \text { for } 0 \leq r<\frac{n}{2} .
$$

This proves Theorem 2 .

Theorem 1 follows immediately from Theorem 2 by taking $Q=P(S)$.

\section{Other problems}

The question answered by Theorem 2 has an obvious generalization. Let $C$ be a simplicial complex, pure of dimension $n$, with vertex set $V$ and let $G \subseteq \operatorname{Sym}(V)$ be a group of automorphisms of $C$. Let $C^{0}$ be the collection of all faces of $V$ which do not contain two elements of $V$ from the same orbit.

Question Suppose $C$ is shellable. What can you say about the dimensions $t$ where $H_{t}\left(C^{0}\right)$ is nonzero?

\section{References}

1. H. Cartan and S. Eilenberg, Homological Algebra, Oxford University Press, Oxford, 1956.

2. S. Fischer, "Signed poset homology and $q$-analog Mobius functions," preprint.

3. P.J. Hilton and U. Stammbach, A Course in Homological Algebra, Springer Graduate Texts in Mathematics, Springer-Verlag, 1971

4. V. Reiner, "Signed posets," JCTA 62(2) (1993), 324-360. 